\title{
Movement Prediction-based Routing (MOPR) Concept for Position-based Routing in Vehicular Networks
}

\author{
Hamid Menouar and Massimiliano Lenardi \\ HITACHI Europe SAS \\ Sophia Antipolis, France \\ \{Hamid.menouar, Massimiliano.Lenardi\}@hitachi-eu.com
}

\author{
Fethi Filali \\ Institut Eurecom \\ Sophia Antipolis, France \\ Fethi.Filali@eurecom.fr
}

\begin{abstract}
Nowadays, researchers show more and more interests to Vehicular Ad hoc NETworks (VANETs), which are a specific instance of Mobile Ad hoc NETworks (MANETs) where nodes are vehicles. In VANETs, vehicles have no energy resource constraint which could extend coverage and network lifetime, but have a high mobility patterns that cause frequent and fast topology changes. Consequently, VANETs have particular research interests, like dedicated $\mathrm{MAC}$ and routing optimization. In our previous work, we have proposed MOvement Predictionbased Routing (MOPR) concept for VANETs, which improves the routing process by selecting the most stable route in terms of lifetime with respect to the movement of vehicles. And in this paper, we present how this MOPR concept can be applied to position-based routing protocols, and how it improves their performances. Based on simulation results we compare MOPR with the position-based routing protocol GPSR and another movement-based routing protocol called MORA.
\end{abstract}

Keywords-Geo-routing, movement-based routing, position-based routing, VANET.

\section{INTRODUCTION}

$\mathrm{I}^{\mathrm{r}}$ $\mathrm{N}$ Vehicular Ad-hoc Networks (VANETs) almost all nodes are vehicles. This makes available more storage and power on each node, thus wider transmission ranges and longer lifetimes are possible [1]. Since nodes are vehicles, the movement speed in such kind of networks is relatively high, which causes frequent and fast network topology changes, and further instability of the wireless communication channel. This makes existing and well working MANETs routing protocols not suitable (as they are) for VANETs [2]-[3].

VANET's researchers should face these problems when proposing new technical solutions, like data routing and shared resources access protocols.

One important advantage of VANETs is that each vehicle, by using a positioning system like GPS or GALILEO, is able to get its own geographic position, thus its movement information (position, speed, movement direction). One more advantage in VANETs is the non-random mobility of the vehicles; roads are mapped and digitally available, and driving rules can be electronically represented as well.

The applications for VANETs can be roughly divided into two main categories: the ones related to active safety on the roads ${ }^{1}$, and the applications dedicated to improve the drivers and/or passengers comfort.

We address here the second category, where most of the time a routing protocol is needed, in order to deliver relatively huge data to a particular destination in real time over multihop paths. On the contrary, for active safety applications, information (generally small) should be provided to all surrounding vehicles in most cases, so a broadcast forwarding protocol is needed.

In previous works [4]-[5] we have proposed a new concept named MOPR (MOvement Prediction Routing) to improve and adapt existing unicast routing protocols and make them suitable for VANETs. This concept, based on vehicles movement prediction, estimates the stability of each communication link in the network in terms of communication lifetime, and then selects the most stable route composed by the most stable intermediate links from the source till the destination. We have integrated MOPR in Ad hoc On Demand Distance Vector (AODV) [6] using the network simulator NS2 [7] and showed its performance improvements.

In this paper, we investigate how MOPR can be applied to position-based routing protocols. We propose a new MOPRbased heuristic for the next hop selection in position-based routing. We have applied this MOPR-based heuristic to the well known position-based routing protocol: Greedy Perimeter Stateless Routing (GPSR) [9]. We have then implemented this MOPR-based GPSR under the network simulator NS2. Some simulation results comparison are given in this paper, they show the MOPR performances comparing those of both basic GPSR and MOvement-based Routing Algorithm (MORA) [10], which is another movement-based routing protocol.

The remainder of this paper is organized as follows: Section II overviews the MOPR concept and it application over unicast routing. Section III gives a short overview on GPSR and MORA. In Section IV we present how MOPR can be applied to position-based routing protocols, and in Section $\mathrm{V}$ some interesting simulation results are given with comparing MOPR performances against the basic GPSR and MORA.

${ }^{1}$ Active because trying to inform the drivers and/or to act on the vehicle in order to avoid accidents instead of alleviating their consequences like airbags. 
Finally, Section VI concludes this paper and outlines future work.

\section{MOPR OVERVIEW}

MOPR determines the most stable path from a source to a destination in terms of communication lifetime by selecting the most stable intermediate links, then, the best intermediate vehicles. For example, assuming we have a network protocol which is capable to provide several unicast paths to a destination, one of those paths can result to be more stable with respect to the others. A stable path can increase the probability that link failures will be avoided during the whole communication. MOPR, based on vehicles' movement information, guarantees the selection of the best next hop for data forwarding. Using MOPR, each vehicle estimates the Link Stability (LS) for each neighboring vehicle before selecting the next hop for the data forwarding/sending. The LS is a relation between the link communication lifetime and a constant value (say: $\sigma$ ) which represents in general cases the routing route validity time, and it depends on the used routing protocol. Figure 1 shows how link lifetimes are estimated based on neighbors' movement information. The lifetime of the link (i, j) (LifeTime $[i, j]$ ) corresponds to the estimated time $\Delta t=t_{1}-t_{0}$ with $t_{1}$ is the time when $D_{1}$ becomes equal or bigger than the communication range $\mathrm{R}$ (i.e. the time when $\mathrm{j}$ goes out of the communication rage of i). $D_{1}$ and $\Delta t$ are estimated using the initial positions of $\mathrm{i}$ and $\mathrm{j}\left(\left(X_{i 0}, Y_{i 0}\right)\right.$ and $\left(X_{j 0}, Y_{j 0}\right)$, and their initial speeds $\vec{V}_{i}$ and $\vec{V}_{j}$ respectively).

$D_{1}^{2}=\left(\left(X_{i 0}+V x_{i} \Delta t\right)-\left(X_{j 0}+V x_{j} \Delta t\right)\right)^{2}+$

$\left(\left(Y_{i 0}+V y_{i} \Delta t\right)-\left(Y_{j 0}+V y_{j} \Delta t\right)\right)^{2}$

$D_{1}^{2}=A \Delta t^{2}+B \Delta t+C$

with : $\left\{\begin{aligned} A= & \left(V x_{i}-V x_{j}\right)^{2}+\left(V y_{i}-V y_{j}\right)^{2} \\ B= & 2\left[\left(X_{i 0}-X_{j 0}\right)\left(V x_{i}-V x_{i}\right)+\right. \\ & \left.\left(X_{i 0}-Y_{j 0}\right)\left(V y_{i}-V y_{j}\right)\right] \\ C= & \left(X_{i 0}-X_{j 0}\right)^{2}+\left(Y_{i 0}-Y_{j 0}\right)^{2}\end{aligned}\right.$

By solving the equation $A \Delta t^{2}+B \Delta t+C-R^{2}=0$ we can easily find the $\Delta t$ which corresponds to the Life Time $[i, j]$ we are looking for.

Now, LS is calculated as follows:

$L S[i, j]=\frac{\text { LifeTime }[i, j]}{\sigma}$, with $L S[i, j]=1$ when

\section{LifeTime $[i, j] \geq \sigma$}

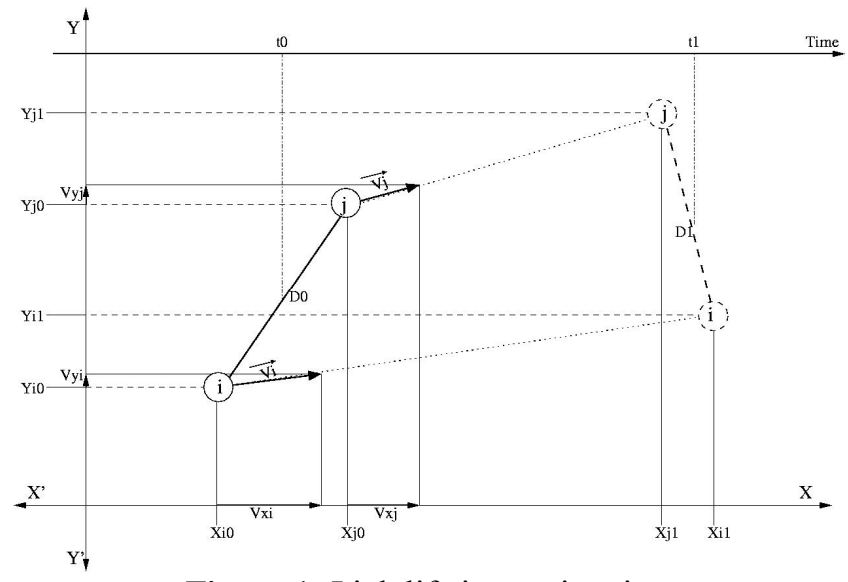

Figure 1: Link lifetime estimation.

Once LS is calculated for each neighboring vehicle, MOPR selects as a next hop for data forwarding/sending the one corresponding to the highest LS (corresponding to the most stable neighboring link).

This approach should help as well in minimizing the risk of broken links and in reducing data loss and link-layer and transport retransmissions.

In [4]-[5] a detailed description of the MOPR concept is provided.

\section{RELATED WORK}

\section{A. A brief overview on GPSR}

Greedy Perimeter Stateless Routing (GPSR) [9] is one well known position-based routing protocol. It uses greedy forwarding to send/forward packets to neighboring nodes that are the closest to the destination. In GPSR it is assumed that every node in the network knows the exact physical location of its neighbors, and that of the destination as well. In regions of the network where such a greedy forwarding is not possible, GPSR recovers by forwarding in perimeter mode, in which a packet traverses successively closer faces of a planar subgraph of the full radio network connectivity graph, until reaching a node closer to the destination, where greedy forwarding resumes.

\section{B. MORA}

To improve the position-based routing performances in VANETs, F. Granelli et all. have proposed in [10] a Movement-Based Routing Algorithm (MORA) for vehicular ad hoc networks. They have applied this algorithm to GPSR. MORA takes into account the physical location of neighboring vehicles and their movement direction when selecting the next hop for sending/forwarding packets. More details about MORA can be found in [10].

We believe that considering only the position and the 
movement direction is not enough for a best next hop selection in VANETs. The vehicles driving speed is important and should be taken into account as well. A vehicle which is almost out the communication range should not be selected as a next hop, which can not be guaranteed without taking into account the speed. In the following section we propose our MOPR concept applied to GPSR, with taking into account neighboring vehicles position and movement direction, and their movement speed as well. Thus, with MOPR vehicle which is estimated to go out the communication range in a short duration time will not be selected as a next hop for data routing if some better candidate is available.

\section{MOPR-BASED GEO-ROUTING}

To show the performance improvements of MOPR over position-based routing protocols we have applied it over GPSR. In the following we present how our MOPR-based GPSR (say MOPR-GPSR) works.

It is not suitable to apply MOPR to GPSR as it is done to unicast routing in our previous works [4]-[5], where MOPR tries to select the path with the longest lifetime. In GPSR, a vehicle dose not save any route to a destination, and do not use the same path for the whole transmission. For each packet to send or to forward, a vehicle selects a next hop among its neighbors. The selected next hop will be used for one packet transfer and then a next hop selection process is done for the next packet to send/forward. When applying MOPR to GPSR as it is, the selected paths should be same or longer in terms of hops number when compared to basic GPSR.

And the calculation of neighboring links' LS before sending/forwarding each packet takes a considerable time. All that decreases the routing performances.

To face this problem, we have applied MOPR in a different way. When a vehicle wants to send or forward data, it first estimates the future geographic location after a duration time $\mathrm{T}$ in seconds for each neighbor. $\mathrm{T}$ is counted in seconds, and it is fixed to $1 \mathrm{~s}$ in our simulations in Section 5. Then, it selects as next hop the closest neighbor to the destination which has not a future location out of its communication rage after the time $\mathrm{T}$.

By doing that, MOPR-GPSR avoids the case when a next hop goes out of the communication range during a data packet transmission. Thus, decreases the data loss and link-layer and transport retransmissions, which increases the routing performances.

\section{SimULATION AND RESULTS}

To evaluate the performances of MOPR over position-based routing protocols, we have implemented it in the NS2.28 network simulator [7] on top-of GPSR. Let us name this implementation MOPR-GPSR. In this implementation, a Hierarchical Location Service (HLS) is used to provide the exact position information of the neighboring and the destination vehicles. More information on HLS is given in [8].

In our simulations we have used a 5000 meters length highway scenario, with 200 vehicles moving on it as shown in Figure 2. In each direction we have three lanes with different speed ranges starting from a minimum speed value of $70 \mathrm{~km} / \mathrm{h}$ and a maximum speed value which we increase from 120 to $220 \mathrm{~km} / \mathrm{h}$. In each direction we have a density of 5 vehicles every $150 \mathrm{~m}$.

We have used the classical 802.11 Medium Access Control (MAC) functionalities, i.e. Distributed Coordination Function (DCF), Carrier Sense Multiple Access with acknowledgments (CSMA/CA with ACK) and Request-To-Send Clear-To-Send (RTS/CTS), and fragmentation, even if we suppose the messages are enough small. Traffic type was CBR with 1024 Bytes of packet size and a 512 bps of maximum CBR rate. One transmitting source and destination vehicles are selected randomly along the middle lane (Normal vehicles' lane) in each direction.

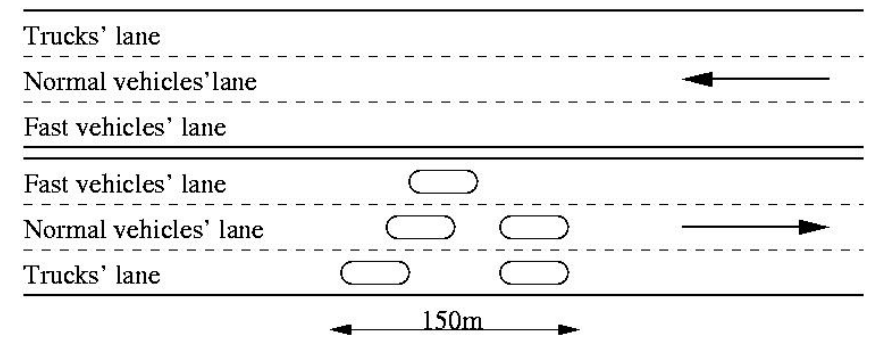

Figure 2: The highway scenario used in our ns2 simulations.

The performances metrics we have studied are as follows:

- packet delivery ratio: defined as the number of correctly received packets at the destination vehicle over the number of packets sent by the source vehicle.

- delay: defined as the average time in seconds that a data packet takes to travel from the source till the destination vehicle.

- routing overhead: defined as the number of bytes injected in the network by the routing protocol.

- routing overhead ratio: defined as the routing overhead caused by the routing protocol over the size of correctly received packets at the destination vehicle.

Figure 3 shows the Packet Delivery Ratio (PDR) obtained for each routing protocol as function of vehicles' maximum speed. It is clearly shown that both MOPR and MORA guarantee a better PDR when compared to basic GPSR. As shown, higher the vehicles' maximum speed, higher the PDR of MOPR when compared to MORA. This means that MOPR guarantees the best PDR when speed is higher. 


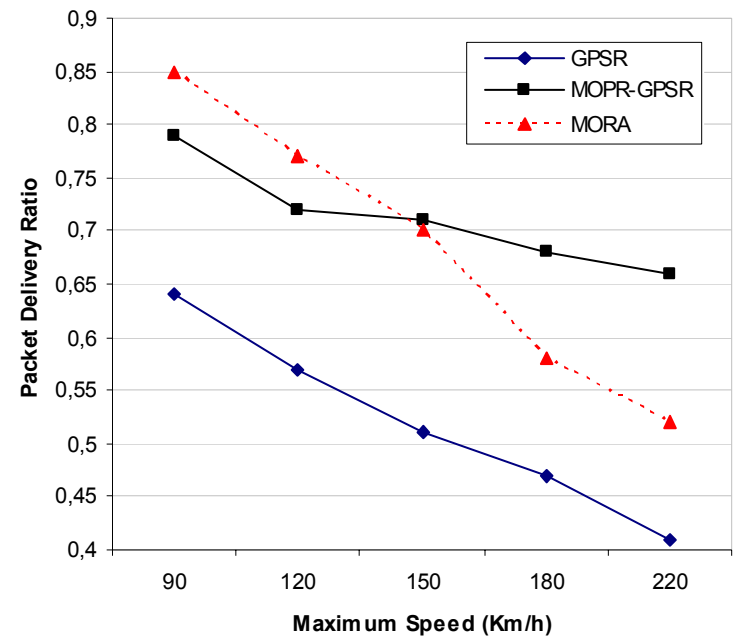

Figure 3: Packet delivery ratio comparison between GPSR, MOPR, and MORA.

Figure 4 shows the delay for each routing protocol as function of vehicles' maximum speed. And as you can see, MOPR improves the delay by at least two times when compared to both basic GPSR and MORA.

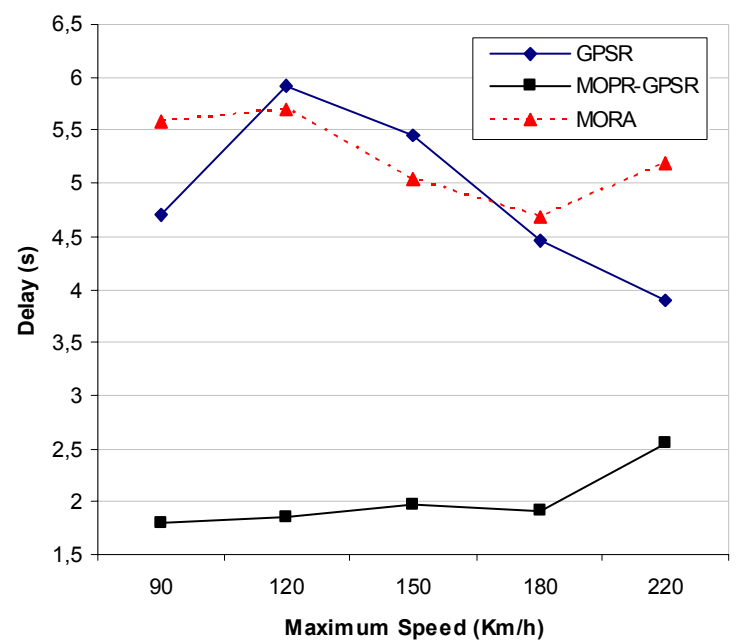

Figure 4: Delay comparison between GPSR, MOPR, and MORA.

Figure 5 shows the routing overhead as function of vehicles' maximum speed. MOPR decreases the routing overhead when compared to basic GPSR, but MORA decreases the routing overhead more. That means that MORA is the best in terms of routing overhead. But, in Figure 6 you can see the Hierarchical Location Service (HLS) overhead caused in the network which should be taken into account to evaluate the real performance of our routing protocols in terms of routing overhead. And it is clearly shown that MOPR is the best in terms of HLS overhead when compared to both basic GPSR and MORA.

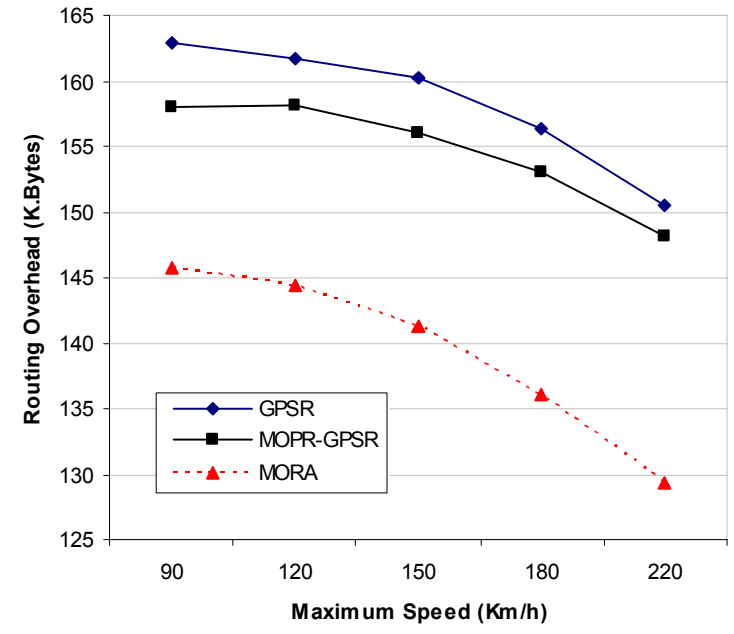

Figure 5: Routing overhead comparison between GPSR, MOPR, and MORA.

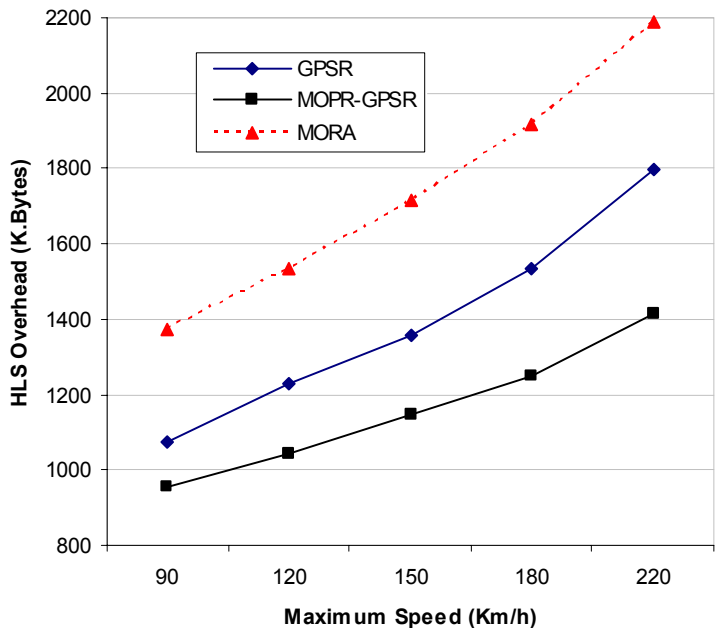

Figure 6: HLS overhead comparison between GPSR, MOPR, and MORA.

To evaluate the performance of any routing protocol in terms of routing overhead, it is important to look to the Routing Overhead Ratio (ROR). Figure 7 shows the ROR caused in our network while taking into account only the routing overhead (i.e. without counting the HLS overhead). It is clearly shown that both MOPR and MORA improve the ROR when compared to basic GPSR. And MOPR shows an almost stable ROR compared to MORA which increases when the maximum speed increases. 


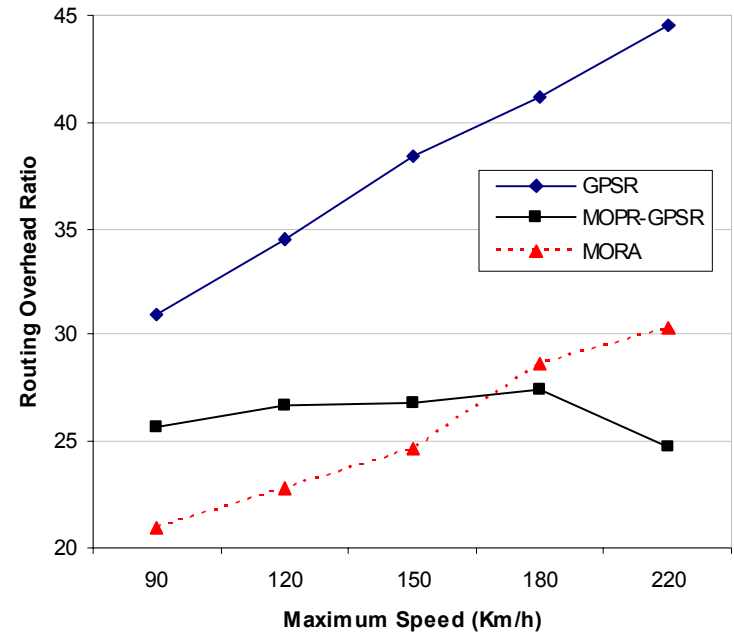

Figure 7: Routing overhead ratio comparison between GPSR, MOPR, and MORA.

The ROR is important, but in such kind of routing protocol, the global ROR overhead, while taking into account the HLS overhead as well, is more important. Figure 8 shows clearly how MOPR improves the network performance in terms of global ROR by about two times when compared to both basic GPSR and MORA.

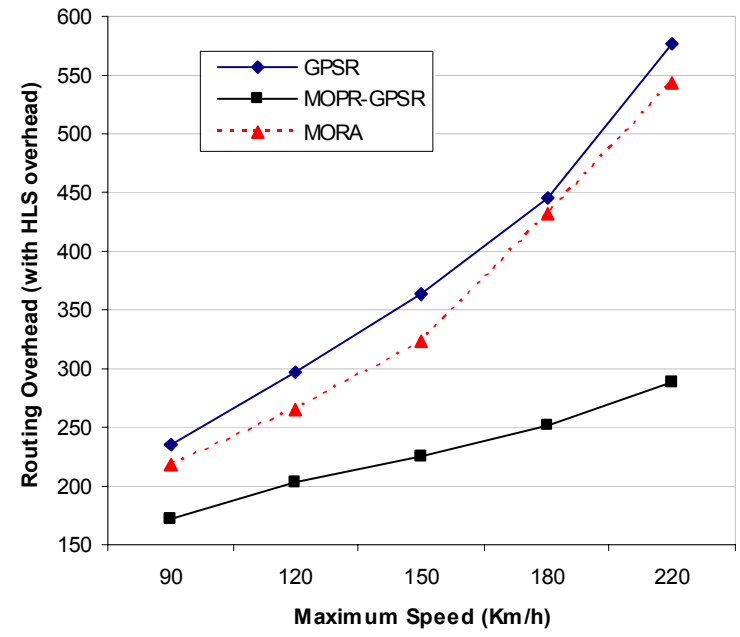

Figure 8: Global routing overhead (routing + HLS) ratio comparison between GPSR, MOPR, and MORA.

All simulation results presented in this section show that MOPR improves the routing performances from all sides. Consequently, MOPR shows a big potential for position-based routing in VANETs.

\section{CONCLUSION}

In VANETs nodes are vehicles, they have the main characteristics of the relative high moving speed. This causes frequent and fast topology changes in the network, which make existing routing protocols basically designed for MANETs not suitable for VANETs.

In some previous works we have proposed MOPR which is an algorithm that, based on node movement information (position, direction, and speed), improves the routing process in MANETs, and mainly in case of high nodes' speed, like in VANETs, by predicting the future neighboring vehicles positions.

After having shown the performance of MOPR to unicast routing protocols in our previous works, we presented in this paper how this MOPR concept can be applied to positionbased routing protocols. For our simulation we have implemented MOPR on top-of the well known position-based routing protocol GPSR.

Very promising ns2 simulation results are presented in this paper; they show that MOPR clearly improves the routing performances when compared to the basic GPSR, and as well when compared to another movement-based routing protocol named MORA, which is based only on position and movement direction.

\section{REFERENCES}

[1] J. Luo and J-P. Hubaux, "A Survey of nter-Vehicle Communication," School of Computer and Communication Sciences, EPFL, CH-1015 Lausanne, Switzerland, Technical Report IC/2004/24, 2004.

[2] J. J. Blum, A. Eskandarian, and L. J. Hoffiman," Challenges of Intervehicle Ad Hoc Networks," in the IEEE Transaction on Intelligent Transportation Systems, Vol. 5, No. 4, December 2004.

[3] C. Lochert, H. Hartenstein, J. Tian, H. Fussler, D. Hermann, M. Mauve, "A Routing Strategy for Vehicular Ad Hoc Networks in City Environments," 58th IEEE Semiannual Vehicular Technology Conference VTC 2003-Fall, pp. 156-161, Orlando, FL, USA, October 2003.

[4] H. Menouar, M. Lenardi, and F. Filali, "A Movement Prediction-based Routing Protocol for Vehicle-to-Vehicle Communications," V2VCOM 2005, 1st International Vehicle-to-Vehicle Communications Workshop, co-located with MobiQuitous 2005, San Diego, California, U.S.A, July 2005.

[5] H. Menouar, M. Lenardi, and F. Filali, "An Intelligent Movement-based Routing for VANETs," ITS World Congress 2006, London, United Kingdom, October 2006.

[6] C. E. Perkins and E. M. Royer, "Ad hoc On-Demand Distance Vector Routing," in the Proceedings of the 2nd IEEE Workshop on Mobile Computing Systems and Applications, pp. 90-100, New Orleans, LA, February 1999.

[7] http://www.isi.edu/nsnam/ns

[8] Wolfgang Kieß, Holger Füßler, Jörg Widmer, and Martin Mauve, "Hierarchical location service for mobile ad-hoc networks," in proceedings of ACM SIGMOBILE Mobile Computing and Communications Review, v. 8, pp. 47-58, 2004.

[9] Karp, B. and Kung, H.T., "Greedy Perimeter Stateless Routing for Wireless Networks," in Proceedings of the Sixth Annual ACM/IEEE International Conference on Mobile Computing and Networking (MobiCom 2000), Boston, MA, August, 2000, pp. 243-254.

[10] F. Granelli, G. Boato, and D. Kliazovich, "MORA: a Movement-Based Routing Algorithm for Vehicle Ad Hoc Networks," IEEE Workshop on Automotive Networking and Applications (AutoNet 2006), San Francisco, U.S.A., December 2006. 